\title{
Bilateral volume reduction in posterior hippocampus in psychosis of epilepsy
}

\author{
James Allebone, ${ }^{\oplus 1,2}$ Richard Kanaan, $^{\oplus, 3}$ Jerome Maller, $^{4,5}$ Terry O'Brien, $_{1}^{6,7}$ \\ Saul Alator Mullen, ${ }^{2}$ Mark Cook, ${ }^{8}$ Sophia J Adams, ${ }^{6}$ Simon Vogrin, ${ }^{9}$ \\ David N Vaughan, ${ }^{2,10}$ Alan Connelly, ${ }^{2,10}$ Patrick Kwan, ${ }^{6,7}$ S F Berkovic, ${ }_{1}^{10}$ \\ Wendyl J D'Souza, ${ }^{11}$ Graeme Jackson, ${ }^{2,10}$ Dennis Velakoulis, ${ }^{12,13}$ Sarah J Wilson ${ }^{1,2,10}$
}

\begin{abstract}
- Additional material is published online only. To view please visit the journal online (http://dx.doi.org/10.1136/ jnnp-2018-319396)
\end{abstract}

For numbered affiliations see end of article.

\section{Correspondence to}

James Allebone, Melbourne School of Psychological Sciences, The University of Melbourne, Melbourne, VIC 3010, Australia; james. allebone@unimelb.edu.au

Received 6 August 2018 Revised 7 December 2018 Accepted 21 January 2019 Published Online First 22 February 2019

\section{Check for updates}

(C) Author(s) (or their employer(s)) 2019. No commercial re-use. See rights and permissions. Published by BMJ.

To cite: Allebone J, Kanaan R, Maller J, et al. J Neurol Neurosurg Psychiatry 2019:90:688-694.

\section{ABSTRACT}

Objective Psychosis of epilepsy (POE) occurs more frequently in temporal lobe epilepsy, raising the question as to whether abnormalities of the hippocampus are aetiologically important. Despite decades of investigation, it is unclear whether hippocampal volume is reduced in POE, perhaps due to small sample sizes and methodological limitations of past research.

Methods In this study, we examined the volume of the total hippocampus, and the hippocampal head, body and tail, in a large cohort of patients with POE and patients with epilepsy without psychosis (EC). One hundred adults participated: 50 with POE and 50 EC. Total and subregional hippocampal volumes were manually traced and compared between (1) POE and EC; (2) POE with temporal lobe epilepsy, extratemporal lobe epilepsy and generalised epilepsy; and (3) patients with POE with postictal psychosis (PIP) and interictal psychosis (IP).

Results Compared with EC the POE group had smaller total left hippocampus volume $(13.5 \%$ decrease, $p<0.001)$, and smaller left hippocampal body $(13.3 \%$ decrease, $p=0.002)$, and left $(41.5 \%$ decrease, $p<0.001)$ and right $(36.4 \%$ decrease, $p<0.001)$ hippocampal tail volumes. Hippocampal head volumes did not differ between groups.

Conclusion Posterior hippocampal volumes are bilaterally reduced in POE. Volume loss was observed on a posteroanterior gradient, with severe decreases in the tail and moderate volume decreases in the body, with no difference in the hippocampal head. Posterior hippocampal atrophy is evident to a similar degree in PIP and IP. Our findings converge with those reported for the paradigmatic psychotic disorder, schizophrenia, and suggest that posterior hippocampal atrophy may serve as a biomarker of the risk for psychosis, including in patients with epilepsy.

\section{INTRODUCTION}

There is a higher prevalence of psychosis in patients with epilepsy compared with the general population. ${ }^{1-7}$ The risk of psychosis of epilepsy (POE) is higher in temporal lobe epilepsy (TLE; 7\%) compared with epilepsy in general $(5.6 \%) .{ }^{8}$ This increased risk, along with the common occurrence of hippocampal sclerosis in TLE, ${ }^{9}$ has led investigators to focus on volume loss in the hippocampus in POE. While some studies have identified reduced hippocampal volumes in POE, ${ }^{10} 11$ others have found no difference relative to epilepsy controls. ${ }^{12-15}$ These conflicting findings likely reflect small sample sizes, methodological limitations and challenges in studying this group. ${ }^{16}$ For example, all studies have either included non-hippocampal tissue such as the choroid plexus ${ }^{17}$ or amygdala, ${ }^{10}$ or excluded posterior hippocampal regions ${ }^{11}{ }^{14}$ in volumetric estimates. No study has separately measured subregions of the hippocampus (head, body and tail) in POE.

The hippocampus is a heterogeneous structure with distinct regional connectivity along its longitudinal axis. Animal studies have shown that it is organised along a gradient from head to tail, ${ }^{18} 19$ superimposed on discrete functional domains ${ }^{20}$ revealed by gene expression studies. ${ }^{21}$ In humans, anterior hippocampal regions show stronger connectivity with the anterior mesial temporal lobe, whereas posterior regions show stronger connectivity with subcortical regions, ${ }^{22}$ which are implicated in dopamine regulation. ${ }^{23-25}$ Altered dopamine regulation is arguably the current leading model of the neuropathogenesis of psychosis, ${ }^{26-28}$ with converging evidence from animal and human studies suggesting that the hippocampus modulates the 'gain' of the dopamine signal. ${ }^{23}$ Atrophy in the posterior relative to the anterior hippocampus has been identified in schizophrenia, ${ }^{29}$ perhaps reflecting abnormal modulation of dopamine signalling by the posterior hippocampus. Separate measurement of posterior hippocampal subregions may thus provide insights into the neuropathogenesis of other psychotic disorders, including POE.

We retrospectively and prospectively collected T1-weighted MRI scans of patients with POE and non-psychotic epilepsy controls (EC) from the three Comprehensive Epilepsy Programs in Melbourne, Australia. Using a protocol that includes $100 \%$ of hippocampal volume, we systematically measured the total hippocampus and the hippocampal head, body and tail bilaterally, hypothesising that (1) compared with EC patients, patients with POE would display significantly smaller hippocampal body and tail volumes bilaterally, and (2) there would be no significant difference in hippocampal head volumes. We also compared hippocampal head, body and tail volumes between patients with POE with TLE, extratemporal lobe epilepsy (ETLE) and genetic generalised epilepsy (GGE) to examine 
whether volumetric changes were related to the epilepsy syndrome or seizure focus. Finally, we compared hippocampal head, body and tail volumes between patients with postictal psychosis (PIP) and interictal psychosis (IP) to assess whether reduced hippocampal volumes differ between these two main POE subtypes.

\section{MATERIALS AND METHODS \\ Experimental design}

This study comprised retrospective and prospective aspects. Retrospectively, in order to identify patients with POE, neuropsychiatrists and epileptologists from each site reviewed the files of all patients who had been admitted to the centres at The Royal Melbourne Hospital, Austin Health and St Vincent's Hospital Melbourne, between January 1993 and September 2014. Inclusion criteria for the POE group were (1) presence of a psychotic disorder and (2) diagnosis of epilepsy based on clinical features and scalp videoelectroencephalogram (EEG) recording of seizures. The specific relationship between the patient's epilepsy and psychotic disorder was established according to the proposed criteria of the International League Against Epilepsy Commission on Psychobiology of Epilepsy. ${ }^{30}$ Accordingly, postictal psychosis was defined as a psychotic episode occurring within 7 days of a seizure or cluster of seizures, after a lucid interval of no more than 48 hours, and lasting no longer than 2 weeks. Interictal psychosis was classified as a psychotic episode temporally independent of seizures (ie, occurring beyond 7 days). Patients were excluded if they (1) had psychogenic non-epileptic seizures; (2) experienced psychotic symptoms as part of ictal semiology only; (3) had medication (including antiepileptic medication) or substance-induced psychoses; (4) had an organic illness (in addition to epilepsy) with known psychiatric symptoms; or (5) if there was no T1-weighted $1.5 \mathrm{~T}$ or $3 \mathrm{~T}$ MRI scan available. Fifty-one patients with POE met the inclusion criteria. The characteristics of each scan in the retrospective cohort are presented in online supplementary table 1 .

Prospectively, patients with POE were identified at the three centres between January 2015 and February 2017. The same inclusion, exclusion and diagnostic procedures applied. Twelve patients with POE were prospectively recruited and scanned on a $3 \mathrm{~T}$ Siemens Trio at the Melbourne Brain Centre, Australia, with a T1-weighted magnetisation-prepared rapid gradient echo image acquired $(\mathrm{TR}=1900 \mathrm{~ms}$, inversion time $=900 \mathrm{~ms}, \mathrm{TE}=2.6$ $\mathrm{ms}$, acquisition matrix $=256 \times 256$, flip angle $=9,0.9 \mathrm{~mm}$ isotropic voxels). Participants with POE were classified as TLE or ETLE based on the location of the seizure focus, determined by scalp video-EEG and MRI, or as GGE if the EEG showed characteristic generalised (bilateral) spike-wave discharges with consistent symptoms. All 63 MRI scans were reviewed by JA and excluded if scan contrast precluded accurate hippocampal tracing. To ensure reliability, a subset of scans $(n=6)$ were also independently reviewed by JM, with $100 \%$ agreement between the two raters regarding exclusion. Thirteen scans were excluded due to poor contrast. The final POE group $(\mathrm{n}=50)$ comprised 28 patients with PIP and 22 with IP.

Epilepsy controls were identified via the same file review process, and following review for image quality were matched as closely as possible to a participant with POE on the following variables: age ( \pm 5 years), gender, epilepsy syndrome, laterality and lesion status on MRI. The inclusion criteria for the EC group were (1) a diagnosis of epilepsy based on clinical features and scalp video-EEG recording of seizures, and (2) no history of psychotic disorder. In order to control for scanner variance, EC participants were selected that had been scanned on the same MRI scanner as their POE counterpart. A flow diagram of the recruitment, screening and matching process is presented in figure 1.

\section{MRI analyses}

All MRI scans were converted to isotropic, rescaled to $1.00 \mathrm{~mm}^{3}$ and anonymised to allow blinded hippocampal tracing. Hippocampal volumes were traced by JA using Analyze V.12.0 (Brain Imaging Resource, Mayo Clinic, Minnesota). Boundaries of the hippocampus were defined according to the EADC-ADNI Harmonized Protocol for Hippocampal Segmentation, which has high measurement reliability ${ }^{31}$ and captures $100 \%$ of hippocampal tissue, including the tail. ${ }^{32}$ Volumes were outlined on consecutive coronal slices in a rostral to caudal direction. Segmentation was regularly checked in the sagittal and axial planes using three-dimensional rotation. The most anterior slice of the hippocampal head was where the hippocampus or the alveus first appears below the amygdala. The most posterior slice was the last slice where the hippocampus appears as a folded structure in the sagittal view, or a double-layered structure in the coronal view. The most anterior portion of the body was the first slice where the hippocampus appears as a single-layered structure in the coronal view. The tail was defined as beginning at the opening of the crus of the fornix in the coronal view and ending as a small grey matter mass inferomedial to the trigone of the lateral ventricle. In patients who had undergone anterior temporal lobectomy $(\mathrm{POE}=5$ right, 3 left; $\mathrm{EC}=2$ right, 1 left), only the non-surgical side was traced.

To determine intrarater reliability, tracing was repeated on five randomly selected scans approximately 6 months after initial tracing. Intrarater reliability was assessed using a two-way random effects, absolute agreement, average-measures intraclass correlation coefficient. Intraclass correlation coefficients were high: hippocampal head right $=0.95$, head left $=0.99$; body right $=0.94$, body left $=0.99$; tail right $=0.88$, tail left $=0.98$. Tracing was also performed blind to group by a second experienced tracer (JM) on five randomly selected scans. Inter-rater reliability was also assessed using a two-way random effects, absolute agreement, average-measures intraclass correlation coefficient. Intraclass correlation coefficients were high: total hippocampus right $=0.98$, total left $=0.96$; head right $=0.99$, head left $=0.93$; body right $=0.97$, body left $=0.98$; tail right $=0.98$, tail left $=0.83$.

SPM V.12 (Wellcome Department of Cognitive Neurology, UK) was used to calculate cerebrospinal fluid (CSF) volume, intracranial volume (ICV; grey matter plus white matter plus $\mathrm{CSF}$ ) and total brain volume (TBV; grey plus white matter). Segmentations were visually inspected to ensure the data were valid. The whole skull was not within the field of view for 9 patients with POE and 4 EC participants, and as the intracranial and total brain matter volumes could not be calculated in these participants they were excluded from those analyses. Patients who had undergone surgery were also excluded from calculation of ICV and TBV.

\section{Demographic and epileptological variables}

The POE and EC groups did not differ significantly on any of the demographic, epileptological, or ICV and TBV variables $(p>0.05$ for all comparisons; table 1), negating the need to enter covariates in subsequent analyses.

\section{Statistical analyses}

Statistical Package for the Social Sciences (SPSS) V.23.0 was employed for all analyses. Data were checked for univariate 


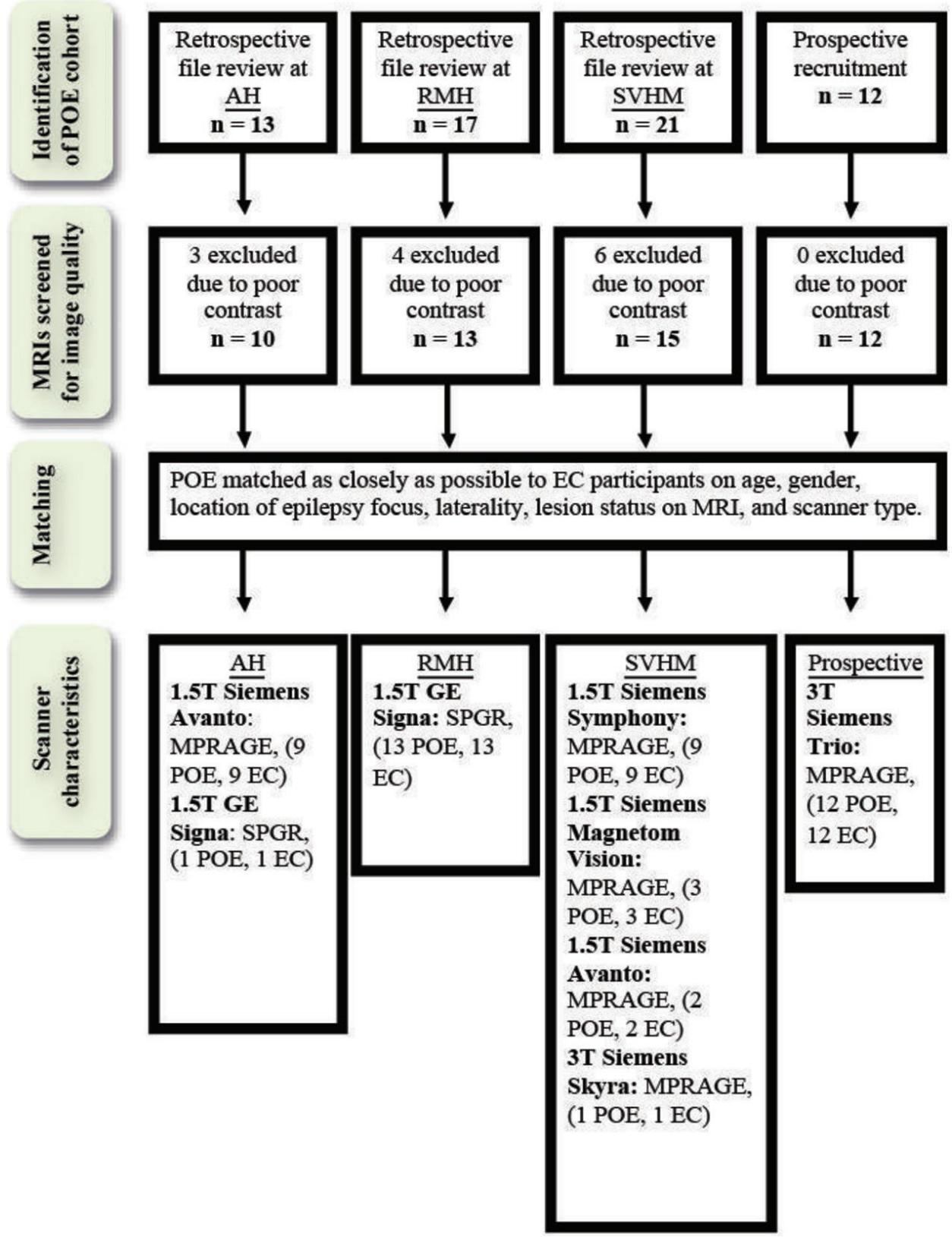

Figure 1 Flow diagram depicting the recruitment, screening and matching process, and scanner characteristics. AH, Austin Health; EC, non-psychotic epilepsy control; MPRAGE, magnetisation-prepared rapid gradient echo; POE, psychosis of epilepsy; RMH, Royal Melbourne Hospital; SPGR, spoiled gradient echo; SVHM, St Vincent's Hospital Melbourne.

normality, univariate and multivariate outliers, homogeneity of covariance matrices, and multicollinearity. Non-parametric tests were employed when assumptions of parametric tests were not met. Two-tailed independent-samples t-tests were used to compare the total hippocampal and segmented head, body and tail volumes in POE relative to EC participants. After Bonferroni correction, the alpha level was $<0.008$, with effect sizes reported. ${ }^{33}$ Although subgroup sizes were small, limiting the interpretability of findings, we conducted an exploratory analysis comparing subregional volumes between patients with POE with TLE, ETLE and GGE, using the Kruskal-Wallis test. One patient with POE with an unclear seizure focus was excluded from this analysis. Total, head, body and tail volumes were compared between PIP and IP groups using independent-samples t-tests. Finally, in order to examine the possible effect of mesial temporal sclerosis on the analysis, pairedsample t-tests were employed to compare the mean ipsilateral and contralateral hippocampal volumes in patients with POE with a known seizure focus $(n=37, \alpha=0.01)$.

\section{RESULTS}

Posterior hippocampal volumes are reduced bilaterally in POE The total mean hippocampal volume was significantly smaller in the POE group on the left, $\mathrm{t}(94)=3.77, \mathrm{p}<0.001, d=0.77$ (medium effect, $13.5 \%$ decrease). The total mean right hippocampal volume was not significantly smaller in the POE group after Bonferroni correction, $\mathrm{t}(91)=2.33, \mathrm{p}=0.022, d=0.48$ (small effect, $8.67 \%$ decrease). There was no significant difference between EC and POE groups in mean hippocampal head volumes on the left, $\mathrm{t}(94)=1.22, \mathrm{p}=0.225, d=0.24$ (small effect, $5.8 \%$ decrease), or right, $\mathrm{t}(91)=0.18, \mathrm{p}=0.856, d=0.03(0.91$ $\%$ increase). Compared with the EC group, the POE group had 
Table 1 Demographic, epileptological and ICV/TBV characteristics of the sample

\begin{tabular}{|c|c|c|}
\hline & POE $(n=50)$ & EC $(n=50)$ \\
\hline \multirow[t]{2}{*}{ Age in years $( \pm S D)$} & $39.80(10.99)$ & $38.64(10.58)$ \\
\hline & Range: $18-64$ & Range: $19-65$ \\
\hline Gender, male, $\mathrm{n}(\%)$ & $29(58)$ & $29(58)$ \\
\hline \multicolumn{3}{|l|}{ Epilepsy syndrome, n (\%) } \\
\hline Temporal & $36(72)$ & $37(74)$ \\
\hline Extratemporal & $9(18)$ & $7(14)$ \\
\hline Generalised & $4(8)$ & $4(8)$ \\
\hline Unclear & $1(2)$ & $2(4)$ \\
\hline \multicolumn{3}{|l|}{ Side of epilepsy focus, $n$ (\%) } \\
\hline Right & $21(46)$ & $23(50)$ \\
\hline Left & $16(34)$ & $16(35)$ \\
\hline Bilateral & $6(13)$ & $5(11)$ \\
\hline Unclear & $3(7)$ & $2(4)$ \\
\hline \multicolumn{3}{|l|}{ Lesion detected on MRI, n (\%) } \\
\hline Lesion positive & $30(60)$ & $23(46)$ \\
\hline Lesion negative & $20(40)$ & $27(54)$ \\
\hline Duration of epilepsy $( \pm S D)$ & $21.53(11.9)^{*}$ & $17.80(12.78) \dagger$ \\
\hline \multicolumn{3}{|l|}{ Intracranial and brain volume } \\
\hline ICV (L) & $1.42(0.17)$ & $1.40(0.15)$ \\
\hline TBV (L) & $1.11(0.11)$ & $1.11(0.19)$ \\
\hline
\end{tabular}

${ }^{*}$ There were 5 cases of missing data.

tThere were 30 cases of missing data.

$E C$, non-psychotic epilepsy controls; ICV, intracranial volume; POE, psychosis of epilepsy; TBV, total brain volume.

a significantly smaller mean left hippocampal body, $\mathrm{t}(94)=3.22$, $\mathrm{p}=0.002, d=0.66$ (medium effect, $13.3 \%$ decrease); left hippocampal tail, $\mathrm{t}(94)=6.47, \mathrm{p}<0.001, d=1.32$ (large effect, $41.5 \%$ decrease); and right hippocampal tail, $\mathrm{t}(91)=6.47, \mathrm{p}<0.001$, $d=1.34$ (large effect, $36.4 \%$ decrease). The right hippocampal body was not significantly smaller in the POE group compared with the EC group, $\mathrm{t}(91)=2.61, \mathrm{p}=0.011, d=0.48$ (small effect, $11.2 \%$ decrease) after Bonferroni correction. All significant results remained significant when patients who had undergone unilateral resection of the hippocampus were excluded from the analysis. Hippocampal volumes for the POE and EC groups are presented in figure 2.

No significant difference in posterior hippocampal volumes in patients with POE with TLE, ETLE and GGE

There was no significant difference between patients with POE with TLE, ETLE or GGE in the total mean hippocampal volume of the left $\left(\chi^{2}[2]=1.20, p>0.05, \eta^{2}=0.02\right)$ or right $\left(\chi^{2}[2]=0.37, p>0.05\right.$, $\left.\eta^{2}=0.00\right)$ sides, or in the left hippocampal head $\left(\chi^{2}[2]=0.76\right.$, $\left.\mathrm{p}>0.05, \eta^{2}=0.00\right)$, body $\left(\chi^{2}[2]=2.07, \mathrm{p}>0.05, \eta^{2}=0.04\right)$ or tail $\left(\chi^{2}[2]=0.75, p>0.05, \eta^{2}=0.01\right)$, or in the right head $\left(\chi^{2}[2]=0.15\right.$, $\left.\mathrm{p}>0.05, \eta^{2}=0.00\right)$, body $\left(\chi^{2}[2]=1.54, \mathrm{p}>0.05, \eta^{2}=0.03\right)$ or tail $\left(\chi^{2}[2]=1.52, p>0.05, \eta^{2}=0.03\right)$. These negative results are difficult to interpret given the sample sizes within subgroups; however, they may suggest that posterior hippocampal atrophy can occur in all epilepsy syndromes. The mean hippocampal volumes in patients with POE with TLE, ETLE and GGE are presented in online supplementary table 2 .

\section{Posterior hippocampal volumes are similarly reduced in PIP and IP}

There were no significant differences between participants with PIP and IP in the total mean volume of the left hippocampus, $\mathrm{t}(47)=0.10, \quad \mathrm{p}>0.05$, or right hippocampus, $\mathrm{t}(44)=1.22$, $\mathrm{p}>0.05$, or in the left hippocampal head, $\mathrm{t}(47)=0.54, \mathrm{p}>0.05$, body, $\mathrm{t}(47)=1.12, \mathrm{p}>0.05$, and tail, $\mathrm{t}(47)=0.89, \mathrm{p}>0.05$, or the right hippocampal head, $\mathrm{t}(47)=0.16, \mathrm{p}>0.05$, body, $\mathrm{t}(44)=0.35$, $\mathrm{p}>0.05$, and tail, $\mathrm{t}(44)=0.71, \mathrm{p}>0.05$. These results suggest that PIP and IP are characterised by similar levels of posterior hippocampal atrophy. The mean hippocampal volumes of the PIP and IP groups are presented in online supplementary table 3 .

Finally, in subjects with POE with a clear side of seizure onset $(n=37 ; 31$ TLE, 6 ETLE), there was no significant difference between the volume of the ipsilateral $(M=1509.58$, $\mathrm{SD}=389.91)$ and contralateral hippocampal head $(\mathrm{M}=1515.62$, $\mathrm{SD}=400.22), \mathrm{t}(64)=0.06, \quad \mathrm{p}>0.01 ; \quad$ or the ipsilateral $(\mathrm{M}=1210.51, \mathrm{SD}=230.39)$ and contralateral hippocampal body $(\mathrm{M}=1218.89, \mathrm{SD}=247.41), \mathrm{t}(64)=0.14, \mathrm{p}>0.01$; or the ipsilateral $(\mathrm{M}=228.44, \mathrm{SD}=82.42)$ and contralateral hippocampal tail $(\mathrm{M}=274.51, \mathrm{SD}=142.38), \mathrm{t}(64)=1.54, \mathrm{p}>0.01$. These results suggest that reduced posterior hippocampal volumes in POE are not underpinned by mesial temporal sclerosis in the POE group.

\section{DISCUSSION}

This study found reduced posterior hippocampal volumes in POE compared with EC participants. Specifically, the hippocampal tail was significantly smaller in POE bilaterally, and the hippocampal body was significantly smaller in the POE group on the left. The results show bilaterally reduced hippocampal volume in POE on a posteroanterior gradient, being most pronounced posteriorly, but still present around the level of the body.

This is the first study to compare subregional hippocampal volumes between a well-matched cohort of POE and EC patients. While some studies have reported reduced hippocampal volumes in POE, ${ }^{10} 11$ sample sizes have been small, with most comprising fewer than 20 participants with POE. Moreover, past studies either did not capture $100 \%$ of the hippocampal tissue, included non-hippocampal tissue or both. For example, some protocols explicitly included the choroid plexus, ${ }^{17}$ while others treated the hippocampus and amygdala as a single structure. ${ }^{10}$ In some studies, all hippocampal volume beyond the last coronal slice on which the uncus is visible was defined as posterior hippocampus, although this region includes both the body and the tail, which have distinct connectivity patterns. ${ }^{22}$ In other studies, the posterior limit of the tail was drawn where the crus of the fornix separates from the fimbria, again excluding a portion of the tail. ${ }^{11} 14$ The striking finding of reduced bilateral posterior hippocampal volume in POE is consistent with studies examining the hippocampus in schizophrenia, where reduced tail volumes, ${ }^{29}$ decreased pyramidal neuronal $\operatorname{size}^{34}$ and density reduction $^{35}$ in the posterior hippocampus have been identified. When considered in light of this research, the results of the present study may suggest an association between posterior hippocampal atrophy and the neuropathogenesis of POE, and perhaps psychosis more generally. Nonetheless, 34 of the patients with POE were scanned after psychosis onset, 4 prior to psychosis onset, with the timing of scanning relative to psychosis onset unclear in the remaining 12 POE cases. Because both duration of psychosis and antipsychotic medication have been linked to altered hippocampal volumes in schizophrenia, these factors may have impacted on hippocampal volumes in the POE group.

Reduced posterior hippocampal volume may be a biomarker of increased risk of POE

Posterior hippocampal volumes in patients with POE with TLE were not significantly smaller than those with ETLE or GGE. While the small numbers of patients in the GGE and ETLE 

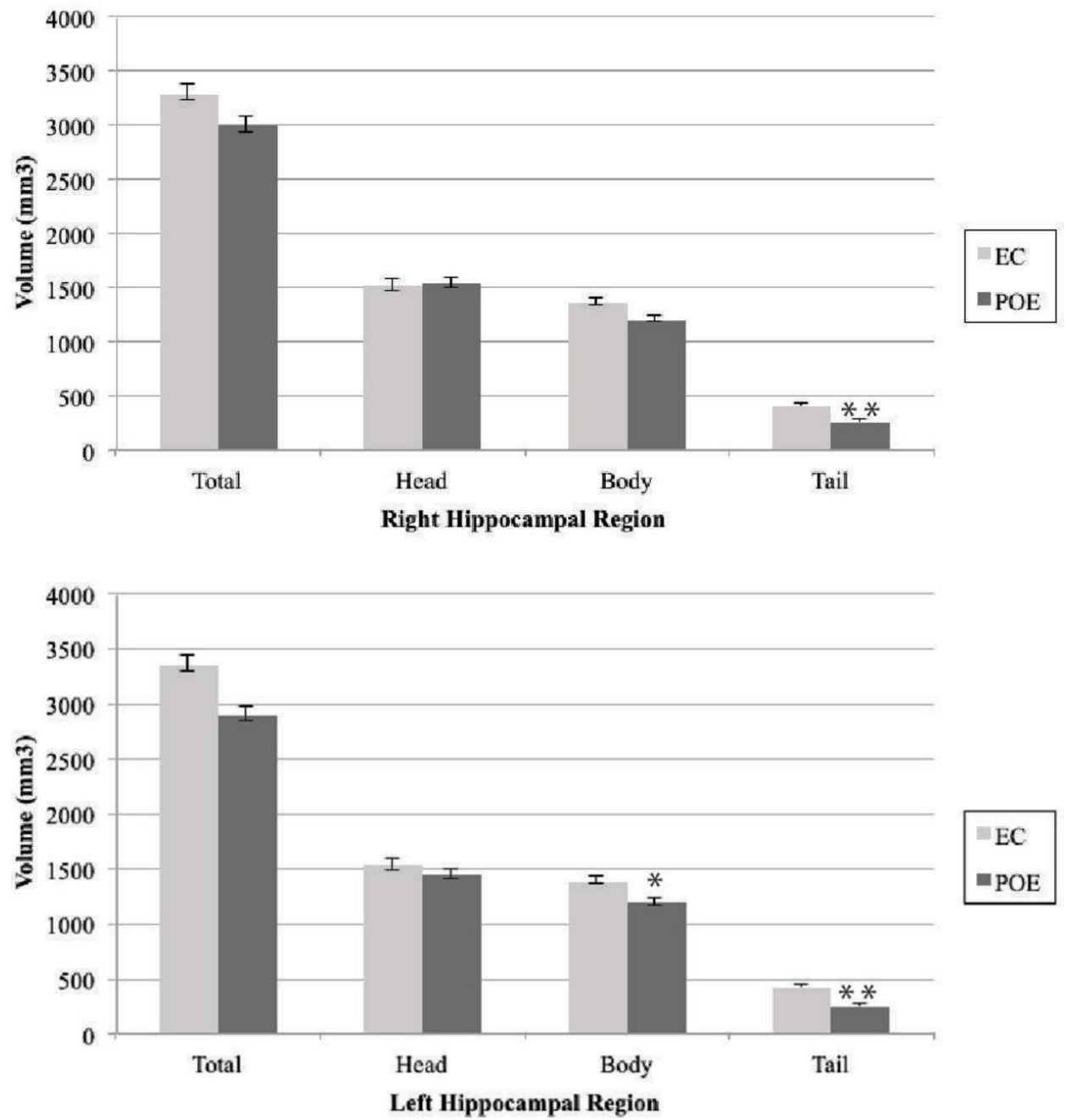

Figure 2 Mean raw hippocampal subregional volumes in the POE and EC groups. Error bars represent $S E M .{ }^{*} P=0.002,{ }^{* *} p<0.001$. EC, non-psychotic epilepsy control; POE, psychosis of epilepsy.

subgroups clearly constrain interpretation of this negative finding, this may suggest that posterior hippocampal atrophy occurs in TLE, and in ETLE and GGE, and may represent a biomarker of increased risk of POE in all patients with epilepsy. While speculative, this possibility is consistent with a growing body of evidence from prevalence studies that challenge the view that there is a specific association between TLE and psychopathology in general, ${ }^{36} 37$ and POE in particular. ${ }^{38}$ The marginally higher prevalence of POE in TLE relative to all other epilepsies $(7 \% \text { vs } 5.6 \%)^{8}$ may just reflect that TLE is the most commonly diagnosed focal epilepsy in epilepsy monitoring units. ${ }^{39}$ The long-held view that TLE carries a higher risk for POE arose from studies reporting a higher rate of POE in TLE relative to other epilepsies, ${ }^{40-42}$ as well as early MRI studies showing abnormalities in mesial temporal lobe structures of patients with schizophrenia, including the hippocampus, amygdala and parahippocampal gyrus. ${ }^{43-46}$ The concept that mesial temporal neuropathology may constitute a biomarker for POE led to a predominance of studies investigating TLE to the exclusion of other types of epilepsy and a focus on mesial temporal structures, particularly the hippocampus. While our findings are supportive of mesial temporal involvement in POE, they do not support the perspective that a temporal lobe seizure focus underpins volumetric changes in this group. However, the small number of patients with GGE and ETLE in our study limits the interpretability of this finding.

Are postictal and interictal psychosis neuropathologically distinct, or points on a continuum of psychosis severity?

While mean hippocampal volumes were smaller in IP compared with patients with PIP in the left hippocampal head and the body and tail bilaterally, these differences did not reach statistical significance. This finding is concordant with the only other study that compared hippocampal volumes in patients with PIP and IP; however, hippocampal subregions were not separately measured. ${ }^{14}$ It may be that future studies with larger sample sizes acquiring data at higher image resolution and with more advanced methods will identify volumetric differences between PIP and IP in the hippocampus. Alternatively, PIP and IP may represent points on a continuum of psychosis severity, mediated by interactions between genetic and/or environmental risk factors, and structural neurobiological change. ${ }^{26}$ The suggestion of a psychosis continuum in POE is supported by evidence for a progression from PIP to $\mathrm{IP}^{47}$ and research highlighting that transitory psychosis in schizophrenia (similar to PIP) can become chronic (similar to IP) depending on the degree of exposure to environmental risk factors, such as childhood trauma, ${ }^{48}$ living in an urban environment ${ }^{49}$ and migrant status. ${ }^{50}$ We suggest that 
future research investigating differences in brain structure and function in PIP and IP incorporate genetic and environmental variables in order to determine the role of mediators of POE severity along a psychosis continuum.

Limitations of this study include our mix of retrospective and prospective recruitment, and the use of multiple MRI scanners. Also, many of the scans were acquired at $1.5 \mathrm{~T}$, and a higher resolution scan may have resulted in more accurate measurement of hippocampal volumes. Nonetheless, scanner variance was controlled for by matching participants with POE to EC participants who had been scanned on the same MRI scanner. Patients were also assessed by different epileptologists and psychiatrists at the time of initial diagnosis. However, clinical and diagnostic information was jointly reviewed by a neuropsychiatrist and epileptologist at each site using a standardised approach, thereby minimising heterogeneity in the final diagnosis.

\section{CONCLUSION}

This study identified bilateral hippocampal volume reduction in patients with POE relative to patients with epilepsy without psychosis. This reduction is moderate at the level of the body and more severe towards the tail. Although the mechanism underpinning reduced posterior hippocampal volume in POE is unclear, our primary finding suggests that smaller posterior hippocampal volumes may be a risk factor for POE. Our finding that patients with PIP and IP display similarly reduced posterior hippocampal volume perhaps suggests that posterior hippocampal atrophy may be a risk factor for both, and that other developmental, neurobiological, genetic or environmental variables mediate the severity of psychosis in people with epilepsy.

\footnotetext{
Author affiliations

'Melbourne School of Psychological Sciences, University of Melbourne, Melbourne, Victoria, Australia

${ }^{2}$ The Florey Institute of Neuroscience and Mental Health, Parkville, Victoria, Australia ${ }^{3}$ Department of Psychiatry, Austin Health, University of Melbourne, Melbourne, Victoria, Australia

${ }^{4}$ ANU College of Health and Medicine, Australian National University, Canberra, Victoria, Australia

${ }^{5}$ Monash Alfred Psychiatry Research Centre, The Alfred and Monash University, Melbourne, Australia

${ }^{6}$ Royal Melbourne Hospital, Melbourne, Victoria, Australia

${ }^{7}$ Department of Neuroscience, Alfred Hospital, Monash University, Melbourne,

Australia

${ }^{8}$ Graeme Clark Institute, University of Melbourne, Melbourne, Australia

${ }^{9}$ St Vincent's Hospital, Melbourne, Victoria, Australia

${ }^{10}$ Comprehensive Epilepsy Program, Austin Health, University of Melbourne, Victoria, Australia

${ }^{11}$ Department of Medicine, St Vincent's Hospital, The University of Melbourne, Australia

${ }^{12}$ Neuropsychiatry Unit, Royal Melbourne Hospital, Melbourne, Victoria, Australia

${ }^{13}$ Melbourne Neuropsychiatry Centre, University of Melbourne and Melbourne Health, Melbourne, Australia
}

\section{Acknowledgements We extend our gratitude to the patients of the} Comprehensive Epilepsy Programs at Austin Health, The Royal Melbourne Hospital and St Vincent's Hospital Melbourne for their time and participation in this research. We also thank the staff of the Programs for their ongoing support.

Contributors JA: study concept and design, acquisition of data, analysis and interpretation of data, drafting and critical revision of the manuscript for intellectual content. RK, JM, SJW, TOB, SAM, MC, SJA, SV, DV, AC, PK, SFB, WDS, GJ and SV: study concept and design, critical revision of the manuscript for intellectual content.

Funding JA is supported by an Australian Postgraduate Award.

Competing interests None declared.

Patient consent for publication Not required.

Ethics approval Both study arms were approved by the Human Research Ethics Committees at The Royal Melbourne Hospital, Austin Health and St Vincent's Hospital
Melbourne, Australia. All participants in the prospective arm of the study provided informed consent.

Provenance and peer review Not commissioned; externally peer reviewed.

\section{REFERENCES}

1 Gudmundson G, Gudmundson G. Epilepsy in Iceland. A clinical and epidemiological investigation. Acta Neurol Scand 1966;43:1-124.

2 Gibbs EL, Gibbs FA, Fuster B. Psychomotor epilepsy. Arch Neurol Psychiatry 1948;60:331-9.

3 Slater E, Beard AW. The schizophrenia-like psychoses of epilepsy: I. Psychiatric aspects. BrJ Psychiatry 1963;109:95-112.

4 Schmitz B, Wolf P. Psychosis in epilepsy: frequency and risk factors. J Epilepsy 1995;8:295-305

5 Jensen I, Larsen JK. Follow-up of 74 patients after resection of a temporal lobe. J Neurol Neurosurg Psychiatry 1979;42:256-65.

6 McKenna PJ, Kane JM, Parrish K. Psychotic syndromes in epilepsy. Am J Psychiatry 1985;182:895-904.

7 Gaitatzis A, Trimble MR, Sander JW. The psychiatric comorbidity of epilepsy. Acta Neurol Scand 2004;110:207-20.

8 Clancy MJ, Clarke MC, Connor DJ, et al. The prevalence of psychosis in epilepsy; a systematic review and meta-analysis. BMC Psychiatry 2014;14.

9 Blümcke I. Neuropathology of focal epilepsies: a critical review. Epilepsy Behav 2009;15:34-9.

10 Maier M, Mellers J, Toone B, et al. Schizophrenia, temporal lobe epilepsy and psychosis: an in vivo magnetic resonance spectroscopy and imaging study of the hippocampus/amygdala complex. Psychol Med 2000;30:571-81.

11 Marchetti RL, Azevedo D, de Campos Bottino CM, et al. Volumetric evidence of a left laterality effect in epileptic psychosis. Epilepsy Behav 2003;4:234-40.

12 Briellmann RS, Kalnins RM, Hopwood MJ, et al. TLE patients with postictal psychosis: mesial dysplasia and anterior hippocampal preservation. Neurology 2000;55:1027-30

13 Marsh L, Sullivan EV, Morrell M, et al. Structural brain abnormalities in patients with schizophrenia, epilepsy, and epilepsy with chronic interictal psychosis. Psychiatry Res Neuroimaging 2001;108:1-15.

14 Tebartz Van Elst L, Baeumer D, Lemieux L, et al. Amygdala pathology in psychosis of epilepsy: a magnetic resonance imaging study in patients with temporal lobe epilepsy. Brain 2002;125:140-9.

15 Flügel D, Cercignani M, Symms MR, et al. A magnetization transfer imaging study in patients with temporal lobe epilepsy and interictal psychosis. Biol Psychiatry 2006;59:560-7.

16 Allebone J, Kanaan R, Wilson SJ. Systematic review of structural and functional brain alterations in psychosis of epilepsy. J Neurol Neurosurg Psychiatry 2018;89:617.

17 Cook MJ, Fish DR, Shorvon SD, et al. Hippocampal volumetric and morphometric studies in frontal and temporal lobe epilepsy. Brain 1992;115:1001-15.

18 Amaral DG, Witter MP. The three-dimensional organization of the hippocampal formation: a review of anatomical data. Neuroscience 1989;31:571-91.

19 Kjelstrup KB, Solstad T, Brun VH, et al. Finite scale of spatial representation in the hippocampus. Science 2008;321:140-3.

20 Strange $B A$, Witter MP, Lein ES, et al. Functional organization of the hippocampal longitudinal axis. Nat Rev Neurosci 2014;15:655-69.

21 Thompson CL, Pathak SD, Jeromin A, et al. Genomic anatomy of the hippocampus. Neuron 2008;60:1010-21.

22 Qin S, Duan X, Supekar K, et al. Large-scale intrinsic functional network organization along the long axis of the human medial temporal lobe. Brain Struct Funct 2016;221:3237-58.

23 Grace AA. Dopamine system dysregulation by the hippocampus: implications for the pathophysiology and treatment of schizophrenia. Neuropharmacology 2012;62:1342-8

24 Floresco SB, Todd CL, Grace AA. Glutamatergic afferents from the hippocampus to the nucleus accumbens regulate activity of ventral tegmental area dopamine neurons. J Neurosci 2001;21:4915-22.

25 Floresco SB, West AR, Ash B, et al. Afferent modulation of dopamine neuron firing differentially regulates tonic and phasic dopamine transmission. Nat Neurosci 2003;6:968-73.

26 Howes $\mathrm{OD}$, Kapur $\mathrm{S}$. The dopamine hypothesis of schizophrenia: version III--the final common pathway. Schizophr Bull 2009;35:549-62.

27 Davis KL, Kahn RS, Ko G, et al. Dopamine in schizophrenia: a review and reconceptualization. Am J Psychiatry 1991;148:1474-86.

28 van Rossum JM. The significance of dopamine-receptor blockade for the mechanism of action of neuroleptic drugs. Arch Int Pharmacodyn Ther 1966;160.

29 Maller JJ, Daskalakis ZJ, Thomson RHS, et al. Hippocampal volumetrics in treatmentresistant depression and schizophrenia: the devil's in De-Tail. Hippocampus 2012;22:9-16

30 Krishnamoorthy ES, Trimble MR, Blumer D. The classification of neuropsychiatric disorders in epilepsy: a proposal by the ILAE Commission on Psychobiology of epilepsy. Epilepsy Behav 2007;10:349-53. 
31 Frisoni GB, Jack CR, Bocchetta M, et al. The EADC-ADNI harmonized protocol for manual hippocampal segmentation on magnetic resonance: evidence of validity. Alzheimers Dement 2015;11:111-25.

32 Boccardi M, Bocchetta M, Apostolova LG, et al. Delphi definition of the EADCADNI harmonized protocol for hippocampal segmentation on magnetic resonance. Alzheimers Dement 2015;11:126-38.

33 Cohen JW. Statistical power analysis for the behavioural sciences. Hillside, NJ: Lawrence Erlbaum Associates, 1988.

34 Benes FM, Sorensen I, Bird ED. Reduced neuronal size in posterior hippocampus of schizophrenic patients. Schizophr Bull 1991;17:597-608.

35 Rametti G, Segarra N, Junqué C, et al. Left posterior hippocampal density reduction using VBM and stereological MRI procedures in schizophrenia. Schizophr Res 2007:96:62-71.

36 Adams SJ, O'Brien TJ, Lloyd J, et al. Neuropsychiatric morbidity in focal epilepsy. Br J Psychiatry 2008;192:464-9.

37 Swinkels WA, van Emde Boas W, Kuyk J, et al. Interictal depression, anxiety, personality traits, and psychological dissociation in patients with temporal lobe epilepsy (TLE) and extra-TLE. Epilepsia 2006;47:2092-103.

38 Manchanda R, Schaefer B, McLachlan RS, et al. Interictal psychiatric morbidity and focus of epilepsy in treatment-refractory patients admitted to an epilepsy unit. Am J Psychiatry 1992;149.

39 David A, Fleminger S, Kopelman M, et al. Lishman's Organic Psychiatry. WileyBlackwell: Oxford:, 2010.

40 Flor-Henry P. Psychosis and temporal lobe epilepsy. A controlled investigation. Epilepsia 1969;10:363-95.
41 Trimble MR. The Psychoses of Epilepsy. New York: Raven Press, 1991.

42 Kanemoto K, Tsuji T, Kawasaki J. Reexamination of interictal psychoses based on DSM IV psychosis classification and international epilepsy classification. Epilepsia 2001:42:98-103.

43 Bogerts B, Ashtari M, Degreef G, et al. Reduced temporal limbic structure volumes on magnetic resonance images in first episode schizophrenia. Psychiatry Res Neuroimaging 1990;35:1-13.

44 DeLisi LE, Dauphinais ID, Gershon ES. Perinatal complications and reduced size of brain limbic structures in familial schizophrenia. Schizophr Bull 1988;14:185-91.

45 Young $\mathrm{AH}$, Blackwood $\mathrm{DH}$, Roxborough $\mathrm{H}$, et al. A magnetic resonance imaging study of schizophrenia: brain structure and clinical symptoms. Br J Psychiatry 1991;158:158-64.

46 Breier A, Buchanan RW, Elkashef A, et al. Brain morphology and schizophrenia. A magnetic resonance imaging study of limbic, prefrontal cortex, and caudate structures. Arch Gen Psychiatry 1992;49:921-6.

47 Tarulli A, Devinsky 0, Alper K. Progression of postictal to interictal psychosis. Epilepsia 2001;42:1468-71.

48 Read J, van Os J, Morrison AP, et al. Childhood trauma, psychosis and schizophrenia: a literature review with theoretical and clinical implications. Acta Psychiatr Scand 2005; 112:330-50

49 Krabbendam L, van Os J. Schizophrenia and urbanicity: a major environmental influence--conditional on genetic risk. Schizophr Bull 2005;31:795-9.

50 Cantor-Graae E, Selten J-P. Schizophrenia and migration: a meta-analysis and review. Am J Psychiatry 2005;162:12-24. 